\title{
Factors affecting patient enablement in an Asian setting: a mixed methods study
}

Jason Meng Huey $\underline{\text { Chan }}^{1}$, MBBS, MMed, Andrew Hao Sen Fang ${ }^{1}$, MBBS, MMed, Mitesh $\underline{S h a h}^{1}$, MBBS, MMed

\begin{abstract}
INTRODUCTION Patient-centred medical care has been rising in importance since the turn of the century. It entails treating patients in relation to their biopsychosocial outlook so as to support the management of their conditions. The extent to which a patient is enabled to acquire skills and knowledge can be measured with the Patient Enablement Instrument (PEI) proposed by Howie and colleagues, and it has been noted to be more reflective of a good consultation compared to patient satisfaction scores. This study aimed to determine the level of patient enablement in the Singaporean context and the factors facilitating it.

METHODS We conducted an embedded mixed method study with primary care patients in two phases: (a) a PEI questionnaire was completed by 150 patients; and (b) a qualitative approach using focused group discussions and individual interviews was used to explore factors associated with high enablement.

RESULTS The mean PEI score was $4.5 \pm 4.4$, with significantly higher scores among patients attending specialised primary care clinics. Important physician factors were doctors' advice, attitude and relationship with the patient. Critical system factors included good continuity of care, workload and financial support, while patient factors included their beliefs, preparedness, inquisitiveness and trust, with considerable impact from the influence of community.

CONCLUSION The PEI score in the Singaporean context is similar to that of other Asian contexts, but slightly higher than that reported in Western studies. Good doctor-patient relationships, efficient systems facilitating continuity of care, and motivated and informed patients all contribute to increased enablement.
\end{abstract}

Keywords: doctor-patient relationship, patient enablement, systems efficiency

\section{INTRODUCTION}

Patient-centred medical care has gained importance since the turn of the century, with experts concurring that a patient-centred approach is necessary in order to provide care of the highest quality. ${ }^{(1)}$ This is also in accord with an Institute of Medicine report published in 2001.(2) To provide patient-centred care, patient enablement has been regarded as a vital process. Patient enablement is described as the process by which intervention by a healthcare provider recognises, promotes and enhances a patient's ability to exercise control over his/her health and life. ${ }^{(3)}$ These processes to enhance a patient's ability were seen as important attitudes and events for patients in their ability to understand their conditions as well as their confidence to carry out medical treatment. ${ }^{(4)}$ Studies have also noted that patient enablement correlates with positive quality care outcomes such as asthma control. ${ }^{(5)}$

The Patient Enablement Instrument (PEI), developed from the original work of Howie et al, ${ }^{(6)}$ is currently a widely adopted instrument to measure patient enablement. It comprises six questions (Box 1), each scored on a 3-point scale $(0=$ not applicable/same or less; 1 = better/more; and $2=$ much better/ much more). A maximal score of 12 indicates that the patient has experienced maximal enablement in a consultation. A score of 6 or more is generally taken to indicate that enablement has been achieved. ${ }^{(7)}$

The latest concepts of patient enablement were derived from a review by Fumagalli et $\mathrm{al}^{\left({ }^{(8)}\right.}$ in which the authors

\section{Box 1. Questions in Patient Enablement Instrument:}

As a result of your visit to the doctor today, do you feel you are:

1. Able to cope with life?

2. Able to understand your illness?

3. Able to cope with your illness?

4. Able to keep yourself healthy?

5. Confident about your health?

noted the lack of a clear definition of terms such as patient enablement, empowerment, involvement or engagement, and that these terms often carried overlapping ideas. Upon distinguishing various differences from the literature, Fumagalli et al considered the concept of patient enablement to be a subset of the broader theme of patient empowerment, just that enabled patients were those who had the requisite understanding and knowledge of their conditions and the skills to manage them, although they may not possess the motivation to do so; this motivation was termed 'patient engagement'. The authors also proposed a concept map that patient empowerment could either be a process or an emergent state of achieving self-care, and is broad in nature. Enablement could also be the emergent state of a patient who has gained some knowledge of his or her condition and the confidence to handle it. ${ }^{(8)}$ With the proposed concept map, the various concepts have become clearer, but it remains to be seen if other studies would adopt a similar understanding of the term 'enablement'. 
Certain characteristics of doctors have had positive correlations with patient enablement. Doctors who appear to be more empathetic toward their patients, as measured by the Consultation and Relational Empathy (CARE) measure score, have been able to garner higher levels of patient enablement than colleagues with lower CARE measure scores. ${ }^{(9)}$ A 2002 study showed that of the three factors (doctor's confidence, doctor's empathy and patient's expectations) that correlated with enablement, empathy accounted for $66 \%$ of the variance in a patient's enablement score. ${ }^{(10)}$ Macpherson et al also found a positive correlation between empathy scores and enablement scores, with specific items such as the doctor's ability to listen to a patient, help a patient take control and explain a patient's condition being noted to be aspects of empathy that were significantly correlated to enablement. ${ }^{(11)}$ In a large cross-sectional study of more than 3,000 patients, Mercer et al reported that the perceived empathy of a general practitioner (GP) had a positive effect on patient enablement in both richer and less affluent populations. ${ }^{(9)}$

Besides empathy, a doctor's positive attitude is also associated with greater enablement. It was reported that doctors who approached their patients positively and conveyed an interest in their health and health promotion usually garnered higher patient enablement scores. ${ }^{(12)}$ Health promotion, together with teachings on preventive health, familiarity with the physician, good non-verbal communication, empathy and clinical competency, was found to account for about $72 \%$ of the variance in the PEI. ${ }^{(13)}$ In particular, familiarity with the doctor has been noted to result in higher PEI scores. Howie et al found that doctors in the highest quartile of PEI scores had more patients who knew them better. However, in practices where the consultation time was longer, patients who were new to the practice had slightly lower PEI scores than those who were on regular follow-up. ${ }^{(14)}$ This was not shown to be statistically significant in some studies ${ }^{(10)}$ but was strongly significant in others. ${ }^{(4,15}$ Doctor-patient relationships that focused on a constant, reliable relationship that promoted familiarity were postulated to help doctors offer measures that could change the lives of their patients. ${ }^{(16)}$

Empathy, a positive attitude, health promotion and good non-verbal communication would likely lead to an increased sense of trust in a care provider. Banerjee and Sanyal found that increased trust in a physician was significantly related to increased enablement. ${ }^{(17)}$ Comparing doctors with different training through a cross-sectional quantitative study, Pawlikowska et al found that PEI scores dropped progressively from 4.33 (95\% confidence interval [Cl] 4.09-4.58) for patients seen by family medicine specialists who had been trained in $\mathrm{PEI}$, to $3.44(95 \% \mathrm{Cl}$ $3.21-3.67)$ for family doctors who had not been trained and 3.23 (95\% Cl 2.99-3.47) for general medicine doctors practising in a polyclinic. ${ }^{(18)}$ However, there is a lack of evidence that methods to increase trust in doctors would work, as shown in a 2014 Cochrane review by Rolfe et al that sought to examine whether doctors who had more training garnered higher PEI scores. ${ }^{(19)}$ This could be due to the substantial trust in doctors that already exists, or because the current ways to measure trust lack sensitivity or consistency across studies and populations, which calls for more international studies.
Clinics that provided longer consultations appeared to garner higher PEI scores. Howie and Pawlikowska found that doctors whose patients had higher PEI scores usually had longer consultation times as well as a smaller list of patients to see. ${ }^{(14,18)}$ Howie et al determined that if consultation time was a constant factor, doctors who garnered higher PEI scores could be distinguished as those who provided better care. ${ }^{(6)}$ This could be because a doctor who has a longer consultation would have more time to address a patient's condition and provide more information for management. ${ }^{(20)}$ This had led to many systems using length of consultation as a proxy for consultation quality in view of its relatively easier method of measurement. Some studies have found that when increased time was given for consultation, even in more deprived areas of practice, there was a significant increase in patient enablement. ${ }^{(9)}$ However, other studies have not found such a clear link, ${ }^{(15)}$ although the context of practice (i.e. secondary care settings) in some of these studies afforded a very long consultation time of nearly 55 minutes for new cases and 22 minutes for follow-up care. ${ }^{(21)}$

The setting of a consultation also seemed to affect patient enablement. Patients who had been treated at hospital clinics showed higher PEI scores irrespective of the degree of training of their doctors, compared to patients seen at community clinics. ${ }^{(22)}$ Some studies have shown that the prestige of a hospital and the availability of seemingly better equipment in the hospital setting could directly lead to elevated PEI scores. ${ }^{(23)}$ Surprisingly, another study that tested the effects of after-hours telephone consultation or home visits against treatment at a hospital clinic showed that patients who were seen at hospital clinics were $40 \%$ less likely to be enabled than those who received telephone advice. ${ }^{(24)}$ This is unexpected, as not only was treatment at a hospital centre associated with lower patient satisfaction and enablement, but there was also no difference between the scores of patients who received telephone consultations and home visits. Further studies were suggested to determine the reasons for such differences.

Age of patients was found to be associated with enablement in some studies. Howie et al found that patients who were more than 65 years old had both higher PEI scores and longer consultation times, but did not acknowledge if there were any confounding factors. ${ }^{(14)}$ This finding was similar to that of Mead et al, ${ }^{(25)}$ although studies by Macpherson et al and Mercer et al did not find similar effects, concluding that there was no significant relation between age and PEI scores. ${ }^{(11,21)}$

It has been reported that the patient's psychological state, expectations and disease complexities can also influence enablement. Howie et al reported that patients who did not get a prescription when they had originally wanted one tended to rate their enablement as lower. ${ }^{(14)}$ In the same study, the authors also found that the case mix presented did not affect the overall enablement score, but that more complex cases needed more time to achieve similar PEI scores. However, some studies have found that patients presenting with physical problems alone tended to rate their enablement higher, ${ }^{(25)}$ while those presenting with psychological problems (as measured by the General 
Health Questionnaire) and patients with three or more long-term conditions tended to have poorer PEI scores. ${ }^{(9)}$

Interestingly, although patients who came from an ethnic minority or spoke a different language from the physicians had shorter consultations, they still tended to rate their visit as enabling. ${ }^{(14)}$ Even after controlling for factors such as communication and continuity of follow-up, patients who were ethnic minorities (Blacks, Asians and Chinese) seemed to achieve higher PEl scores. ${ }^{(25)}$ Ozvacić Adzić et al found similar results and proposed that this could be due to differences in these patients' presenting complaints or their acceptance of care given, or simply their different understanding of the PEI concepts and measuring instrument. ${ }^{(15)}$

Earlier studies using the PEI have shown that many patients rate enablement poorly in primary care. A cross-sectional study of more than 3,000 GP consultations in Scotland revealed a mean PEI score of 3 and an even lower median of 2. ${ }^{(9)}$ The reasons for the low scores are still unknown. In the Asian context, experience regarding patient enablement is more limited. From the available studies conducted in Asian populations, the PEI scores tended to be higher than those found in Western studies. A study conducted in India noted that nearly $85 \%$ of respondents felt they were able to cope with their illness, ${ }^{(16)}$ while another study from Hong Kong found a mean PEI score of 4.65. (23) These findings are contrary to common thinking given that in the Asian primary care setting, workloads tend to be heavier, resulting in shorter consultation times.

The objectives of this study were to investigate: (a) how patients with chronic medical conditions rated enablement in a local context; and (b) the factors associated with patient enablement in the local context.

\section{METHODS}

We conducted a mixed method study in a medium-sized polyclinic in Singapore between January and April 2017. The target population consisted of patients who were on follow-up at the polyclinic for at least one chronic medical condition such as diabetes mellitus and hypertension. Participants had to be at least 21 years old and able to give informed consent.

The study was conducted in two phases to address the first and second research questions in sequence. The research incorporated a mixed method approach to allow for the integration of quantitative methods of acquiring patient responses to enablement with their qualitative views. An embedded mixed method approach was used, as the main thrust of the study sought to explore the perceptions of patients, while the quantitative element aids in distinguishing between the range of scores and any relation to the clinic setting. ${ }^{(26)}$ To address the first research question, we used a quantitative method to obtain the PEI scores as a dependent variable in a primary care polyclinic. The hypothesis for this phase was that the patient enablement scores were not different from those found in other Asian countries.

To address the second question, we used a qualitative approach to explore factors associated with high enablement. The phenomenographical approach was used to describe the

\section{Box 2. Semi-structured interview guide:}

1. Can you describe the last consultation that helped you cope with your chronic illness better?

2. Do you remember who helped you to cope better the most? Why?

3. How do you feel healthcare staff can help you obtain skills (e.g. blood pressure monitoring, glucometer) to manage your condition better?

4. What are some of the factors that make a doctor-patient encounter useful?

5. Where do you usually go to look for more information about your health? What makes you go there?

experiences of the patients when they came for primary care consultations. This approach allowed the team to: (a) describe the different experiences of the participants; (b) enhance the richness of the quantitative data by allowing the participants to offer perspectives that had not yet been considered; (c) organise the data into categories; and (d) compare the similarities and differences so as to present them in a systematic way. ${ }^{(7,27)}$

In the first phase, convenience sampling was employed for the estimated required sample size of 138 patients, which was rounded up to 150 patients to account for attrition. Patients were mostly not under the direct care of the researchers. All the investigators (four doctors and one research assistant) were trained in a standardised recruitment protocol. After informed consent was obtained, the patients completed a two-part questionnaire consisting of sociodemographic information and a combined language version of the PEI. The questionnaire was kept anonymous, and either self-completed or assisted by an investigator. All the participants completed the questionnaire on the same day of the consultation in accordance with its original intent. ${ }^{(6)}$

In the second phase, a qualitative approach of focused group discussions (FGDs) and individual interviews (IDIs) was used to explore factors associated with high enablement. Typical sampling was employed to obtain the views and perspectives of a typical patient from the target population. Patients were asked a series of open-ended questions according to a pre-tested, semistructured topic guide (Box 2). A scribe took notes during each discussion, which lasted for an average of 55 minutes. All FGDs and IDIs were audio-recorded.

To analyse the PEI scores in our primary care context, descriptive statistics were presented. As in previous studies, a PEI score of $\geq 6$ was adopted as the threshold for achieving patient enablement. Inferential statistics using the Mann-Whitney $U$ test was applied on variables with two categories, while the Kruskal-Wallis test was applied on variables with more than two categories. Data analysis was performed with IBM SPSS Statistics version 22.0 (IBM Corp, Armonk, NY, USA).

To analyse the qualitative interviews, audio recordings of the IDIs and FGDs were transcribed verbatim by professional transcribers. Field notes were collated and compared with the transcribed data to fill in any gaps in the recordings. After the preliminary exploratory analysis of the data by hand, the data was encoded by examining and labelling segments of data. A code list was made for each transcribed interview and redundant 
codes were eliminated. Codes addressing similar ideas from multiple perspectives were grouped together to form themes. ${ }^{(26)}$ All the investigators agreed with the final codes and themes that emerged. This was an iterative process, which continued until data saturation was reached.

Data triangulation was done through comparison of data from the transcribed notes of both the FGDs and IDIs, as well as comments from participants who could not avail themselves for the interviews. Member checking was done by sending the preliminary themes to one of the participants who had come for the interviews, to determine if he agreed that the themes were representative of the discussion.

\section{RESULTS}

According to the research protocol, 189 patients were approached. The overall response rate was $79.4 \%$, and all the respondents completed the $\mathrm{PEI}$ questionnaire. Wave analysis ${ }^{(26)}$ showed that the patients who were recruited in the first week were not different from those who were recruited during the remaining weeks. The mean age of the patients was $63.50 \pm$ 10.02 (median 62; range 31-88) years. Most of the patients were Chinese $(78.0 \%) .84 .7 \%$ of participants were from the general patient pool, with $12.7 \%$ seen at the family physician clinic (FPC) run by senior doctors and $2.7 \%$ seen by nurse clinicians who co-managed the patient with another doctor. Table I shows the

Table I. Demographics, characteristics and PEI scores of the participants $(n=150)$.

\begin{tabular}{|c|c|c|c|c|c|c|c|}
\hline $\begin{array}{l}\text { Demographic/ } \\
\text { characteristic }\end{array}$ & No. (\%) & PEI score* & p-value & $\begin{array}{l}\text { Demographic/ } \\
\text { characteristic }\end{array}$ & No. (\%) & PEI score* & p-value \\
\hline Age (yr) & & & 0.06 & Clinic seen & & & 0.05 \\
\hline$<60$ & $53(35.3)$ & $5.0(0.5-10.0)$ & & General clinic & $127(84.7)$ & $3.0(0-8.0)$ & \\
\hline$\geq 60$ & $97(64.7)$ & $3.0(0-7.0)$ & & Family physician clinic & $19(12.7)$ & $6.0(2.0-11.0)$ & \\
\hline Gender & & & 0.27 & Others & $4(2.7)$ & $0.5(0-4.0)$ & \\
\hline Male & $88(58.7)$ & $4.0(0-10.0)$ & & Employment status & & & 0.68 \\
\hline Female & $62(41.3)$ & $3.0(0-7.0)$ & & Working & $92(61.3)$ & $4.0(0-9.8)$ & \\
\hline Ethnic group & & & 0.85 & Unemployed & $21(14.0)$ & $3.0(0-9.0)$ & \\
\hline Chinese & $117(78.0)$ & $3.0(0-8.0)$ & & Retired & $37(24.7)$ & $3.0(0-7.0)$ & \\
\hline Malays & $18(12.0)$ & $6.0(0-10.3)$ & & Hypertension & & & 0.57 \\
\hline Indian & $14(9.3)$ & $4.0(0-10.0)$ & & Yes & $114(76.0)$ & $4.0(0-9.0)$ & \\
\hline Eurasian/others & $1(0.7)$ & - & & No & $36(24.0)$ & $3.0(0-8.5)$ & \\
\hline Education & & & 0.11 & Diabetes mellitus & & & 0.16 \\
\hline No formal education & $10(6.7)$ & $2.0(0-5.3)$ & & Yes & $69(46.0)$ & $3.0(0-7.5)$ & \\
\hline Primary & $39(26.0)$ & $2.0(0-6.0)$ & & No & $81(54.0)$ & $4.0(0.5-10.0)$ & \\
\hline Lower secondary/secondary & $61(40.7)$ & $5.0(0-10.5)$ & & Hyperlipidaemia & & & 0.21 \\
\hline $\begin{array}{l}\text { Post-secondary/A-level/ITE/ } \\
\text { diploma }\end{array}$ & $23(15.3)$ & $3.0(0-6.0)$ & & Yes & $116(77.3)$ & $3.0(0-7.0)$ & \\
\hline University/post-tertiary & $17(11.3)$ & $6.0(2.0-11.5)$ & & No & $34(22.7)$ & $4.5(0.8-10.0)$ & \\
\hline Current housing type & & & 0.50 & Ischaemic heart disease & & & 0.61 \\
\hline HDB 1-2-room flat & $14(9.3)$ & $3.0(0-8.3)$ & & Yes & $15(10.0)$ & $4.0(3.0-7.0)$ & \\
\hline HDB 3-4-room flat & $76(50.7)$ & $4.0(0-8.0)$ & & No & $135(90.0)$ & $4.0(0-9.0)$ & \\
\hline HDB 5-room/executive flat & $36(24.0)$ & $3.5(0-10.8)$ & & Chronic kidney disease & & & 0.11 \\
\hline $\begin{array}{l}\text { Condominium/private } \\
\text { property }\end{array}$ & $21(14.0)$ & $2.0(0-6.0)$ & & Yes & $15(10.0)$ & $0(0-6.0)$ & \\
\hline Rental room/apartment & $3(2.0)$ & $10.0(1-11)$ & & No & $135(90.0)$ & $4.0(0-9.0)$ & \\
\hline Medical subsidies & & & 0.06 & Stroke & & & 0.49 \\
\hline CHAS orange or blue card & $35(23.3)$ & $3.0(0-10.0)$ & & Yes & $6(4.0)$ & $1.5(0-7.5)$ & \\
\hline Pioneer Generation card & $47(31.3)$ & $1.0(0-6.0)$ & & No & $144(96.0)$ & $4.0(0-9.0)$ & \\
\hline Others & $8(5.3)$ & $8.0(0-11.5)$ & & COPD & & & 0.92 \\
\hline \multirow[t]{5}{*}{ No subsidies } & $60(40.0)$ & $5.5(1.0-10.0)$ & & Yes & $1(0.7)$ & - & \\
\hline & & & & No & $149(99.3)$ & $4.0(0-9.0)$ & \\
\hline & & & & Asthma & & & 0.89 \\
\hline & & & & Yes & $7(4.7)$ & $6.0(0-6.0)$ & \\
\hline & & & & No & $143(95.3)$ & $4.0(0-9.0)$ & \\
\hline
\end{tabular}

Patient data was obtained from January 2017 to April 2017. *Data presented as median (interquartile range). CHAS: Community Health Assist Scheme; COPD: chronic obstructive pulmonary disease; HDB: Housing and Development Board; ITE: Institute of Technical Education; PEI: Patient Enablement Instrument 
demographics and characteristics of the participants and their PEI scores.

Most of the participants had either hypertension (76.0\%) or hyperlipidaemia (77.3\%). $20.0 \%$ of patients were on follow-up for one main chronic medical condition, while $24.7 \%$, 35.3\%, $13.3 \%, 5.3 \%$ and $4.0 \%$ had two, three, four, five and six chronic medical problems, respectively. Patients with more medical conditions tended to have lower PEI scores, but the relationship was not statistically significant $(p=0.187)$. In addition, patients who were seen at the FPC were significantly more likely to obtain higher PEI scores than patients seen at the general clinic or other clinics (median 6.0, interquartile range [IQR] 2-11; median 3.0, IQR 0-8; and median 0.5, IQR 0-4, respectively, $\mathrm{p}=0.05$ ).

Fig. 1 shows the distribution of the PEI scores. The mean PEI score was $4.5 \pm 4.4$ (range $0-12$ ), while the median score was 4 (IQR 0-9). Of the 150 patients, $40.7 \%$ had a PEI score $\geq 6$ (i.e. considered to be enabled). The prevalence of the floor effect and ceiling effect was $31.3 \%$ and $9.3 \%$, respectively, with the floor effect considered significant at above $15 \% .{ }^{(28)}$ The floor effect indicates that the test used created too many zero scores and could have been too difficult, or in this case, it shows that most of the patients did not have any changes in enablement after their consultation.

Table II shows the themes that emerged from the interviews and the various codes that were used to summarise the participants' statements. Altogether, there were 28 codes, organised into 12 themes. Six IDIs and three FGDs, each consisting of 4-5 participants, were conducted. The interviews revealed three key categories that influenced patient enablement: healthcare staff factors; system factors; and patient factors.

\section{Theme 1: Advice from healthcare staff}

Advice from healthcare staff was frequently cited as one of the ways through which patients were enabled. Patients who attended a healthcare establishment wanted the doctor to explain their conditions using terms that they could understand. This explanation could take the form of specific information about the disease, therapy or targets to achieve, as well as ways to prevent worsening of the disease. Besides simple language that could be easily understood by laymen, patients also appreciated the use of visual aids, where relevant, as graded information instead of a deluge of facts.

"But sometimes I feel it's better for them to have a little bit in black-and-white, as to the nature, and to the cause of the problem, and the remedies for the problem, because as ageing population, (the) tendency for forgetfulness is there." (J34)

\section{Specific information on disease, therapy and targets}

Patients appreciated doctors who spent time advising them on the possible causes, consequences and natural history of their disease. Many participants provided feedback that their doctors did not offer them enough information about the cause of their disease, and some were surprised when told of their diagnoses. Patients also preferred that their doctors revealed to them the severity of their condition; many felt that this was not conveyed when they were first diagnosed.

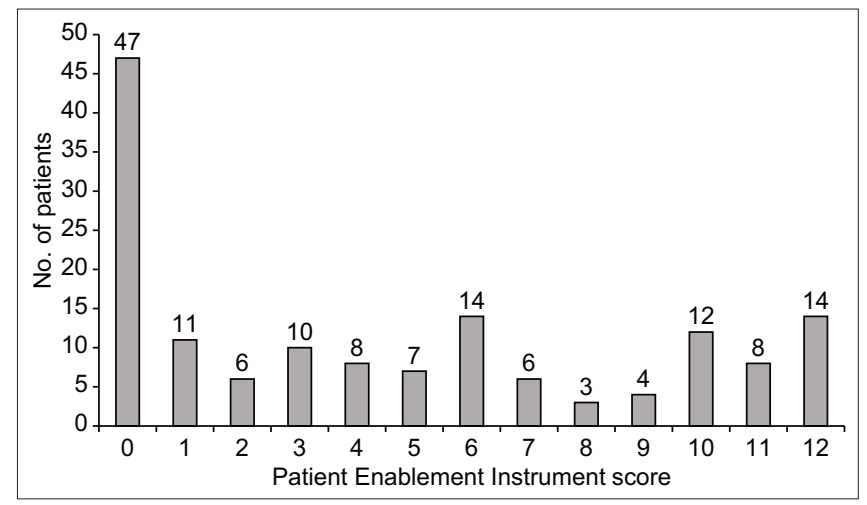

Fig. 1 Graph shows the Patient Enablement Instrument scores of the participants.

"It's a bit sad that a lot of patients who have diabetes, they are not really aware how serious the illness is or diabetes is." (J27) "They said you have to take medicine. That's all. And then there is no other more information, understanding about my illness, what is the cause, how you can improve, etc. And then, after that from there, that's where I struggle." (C2)

Most patients could recount instances when specific advice on therapy, such as lifestyle changes in their diet and exercise, was provided to enable them to make healthier food choices. Some participants recounted specific examples of foods from hawker centres (e.g. chicken rice) that their doctors had advised them to consume sparingly.

"At least is brisk walk, if you cannot do very difficult (exercises), just a brisk walk. And must especially (watch over) food, must diet, really go through a diet." (A2)

Some participants were taught how to achieve various targets in order to have good control over their conditions. They understood the various benchmarks that doctors used and the need to titrate treatment based on these targets. Some were motivated to try their best to see improvements in these targets. To that end, many participants actively engaged in self-monitoring of their home glucose or blood pressure levels, and brought their results to their doctors. However, some participants confessed that even though they had been diabetic for some time, they were not aware of certain self-monitoring devices such as home glucose meters.

"I think generally the readings, they have high and low benchmarks, what the doctor say... is within that range." (C4)

\section{Advice on prevention}

Besides managing the reason for the encounter, patients appreciated doctors who went beyond the reason for their encounter to provide advice on prevention of disease.

"Looking at the reading... good ... all very good ... then she looks at record ... do you come for subsequent tests, other tests. She said, 'You have not checked for hep(atitis), probably book an appointment... do some tests'." (B2)

Health screening tests and preventative measures, such as flu vaccination, were most commonly broached by doctors, and this was seen to enable patients by supporting them in becoming healthier. Some patients expected family physicians to enquire 
Table II. Factors influencing patient enablement: categories, themes and codes that emerged from interviews and focused group discussions.

\begin{tabular}{|c|c|c|}
\hline Category & Theme & Code \\
\hline \multirow[t]{13}{*}{ Healthcare staff factors } & 1. Advice & \\
\hline & \multirow[t]{4}{*}{-What } & 1. Specific disease information \\
\hline & & 2. Specific therapy information (e.g. diet, exercise) \\
\hline & & 3. Targeted treatment (i.e. to know the targets) \\
\hline & & 4. Going beyond (e.g. disease prevention) \\
\hline & -When & 5. Doctor giving graded amount of information \\
\hline & \multirow[t]{2}{*}{$\cdot$ How } & 6. Same language \\
\hline & & 7. Visual aids \\
\hline & \multirow[t]{3}{*}{ 2. Attitude } & 8. Encouragement \\
\hline & & 9. Friendliness \\
\hline & & 10. Concern \\
\hline & \multirow[t]{2}{*}{ 3. Doctor-patient relationship } & 11. Cooperation \\
\hline & & 12. Personalising care \\
\hline \multirow[t]{7}{*}{ System factors } & \multirow[t]{3}{*}{ 4. Continuity of care } & 13. Post-consultation care: checks and reminders from healthcare staff \\
\hline & & 14. Follow through from previous consultation's information \\
\hline & & 15. Team-based care \\
\hline & 5. Image of setting & 16. Good standing among patients, capability \\
\hline & 6. Workload & 17. Heavy workload \\
\hline & 7. Financial aspect & 18. Cost of care \\
\hline & 8. Passive information & 19. Pamphlets and posters \\
\hline \multirow[t]{9}{*}{ Patient factors } & \multirow[t]{5}{*}{ 9. Attitude } & 20. Beliefs \\
\hline & & 21. Mental conditioning \\
\hline & & 22. Inquisitiveness \\
\hline & & 23. Self-motivation \\
\hline & & 24. Trust \\
\hline & \multirow[t]{2}{*}{ 10. Impact of initial care } & 25. Consequences stimulated self-care \\
\hline & & 26. Success breeds success \\
\hline & 11. Community & 27. Community involvement \\
\hline & 12. Actions & 28. Self-help actions needed \\
\hline
\end{tabular}

Categories, themes and codes were obtained from transcribed interviews and codes through an iterative process from six individual interviews and three focused group discussions from January 2017 to April 2017.

about their other medical conditions such as their operations or follow-up appointments at the hospital, as they felt that the management of other conditions seen outside of primary care could affect the treatment provided at the primary care setting.

"For me, it is a major concern. It's almost like, 'Diabetes, okay! I'm doing all right. But the main worry - and it still continues even after fifteen months - (is about) the cancer of my kidney.' How come the doctor never even mentioned anything about it when I come here?" (C1)

\section{Use of common language and aids}

Patients appreciated doctors who could speak their language, as it enabled them to understand their condition and treatment better.

"Because now, doctors are better. Some, because they cannot speak Mandarin or English, they can speak dialect... more easy ... more understanding." (B3)

Other patients supported the use of relevant visual aids, such as photographs from the Internet, or their own laboratory results and trends for key targets, which helped them to understand their condition better. For more complicated skills, such as using an insulin needle for the first time, patients appreciated the use of model kits for demonstration and self-practice.

"How she did was that I think there was a sponge, then she told me, treat this as your stomach, then inject on this. Then she told me to proceed, and I was able to do it." (J27)

\section{Giving graded information}

In terms of receiving information on their conditions, many participants felt that they could only absorb some amount of information at each time. Sometimes, they just wanted to obtain certain 'helpful hints' from healthcare staff, so that they could have a 'better life', but they did not require doctors to send them to a dietician to teach them the basics of diet management.

(Moderator) "And do you feel that that advice and that medication (were) enough, or too little or too much?" "I think it's enough... for the moment." [Laughs] (J42)

Some patients felt that advice could be given at opportune times. By this they meant that they would like to have more 
targeted advice at specific times of the year, for instance before major festivals are celebrated in the country. Being in a multicultural society, local patients enjoyed a wide variety of celebrations, often accompanied by a wide variety of foodstuffs. For example, reminding patients of the calorie contents of various celebratory titbits such as pineapple tarts, which are consumed in high quantities during Chinese New Year, could enable them to reflect on their dietary choices.

(Moderator) "So, it's interesting that you brought up that before Chinese New Year, the doctors highlighted that you need to be more careful. So does it mean that if we tie in our advice to major festivals and holidays in Singapore, that would be more effective?"

"Provided the consultation timing is nearby. Of course that would be, there is a reminder. So it really depends on the timing." (B2)

\section{Theme 2: Doctor's attitude}

\section{Friendliness, encouragement and concern}

Three key attitudes of doctors were brought up during the interviews. Firstly, patients felt more enabled when the doctor was friendly. Sometimes, the warmth of a healthcare staff could help dispel some of the misery and gloom that a patient with chronic disease felt, and that in turn could enable the patient to feel more supported.

"(For) others, they may have other types of sickness, then they have so many sicknesses, I feel that the patients will be very miserable, so they are more or less very 'low spirit' type. Yah, yah! You know, if the patient feel(s) comfortable, and then, you know, it may be easier for the doctor to also speak to the patient and advise him." (C2)

Some patients commented that they would feel 'safe' if the doctor was able to make them feel comfortable, and this could help them to share more about their issues with control. Besides issues with their disease, patients felt that if the doctor could 'click' with them, they would be keener to manage their disease better. This personal touch helped both the doctor and patient see that the patient is not defined by a disease but is a person with hobbies, a job, a family and challenges. This realisation would undoubtedly help the physician to enable the patient more as he customises care that befits the patient.

"Each time he will tell me and we will talk like a friend, more than a patient. So it's very good to have this kind of attitude and this kind of doctor, very patient, listen to you, and share with you. Sometimes you can even share to the extent, talking about our line dancing. You know, that kind of thing, because she does that, so we do that. So it's very good that each time we come, we feel good. Whatever thing that I don't feel good or I don't think this is safe, I will just approach him." (B1)

Patients also cited examples of unfriendly behaviour, such as doctors who projected a 'top-down' attitude, failed to communicate with empathy, or appeared to be more concerned about their data entry into the electronic medical records than conversing with them. In mitigation, participants were hopeful that skills like empathy could be inculcated in doctors who lacked such skills.

"But then if you have a doctor who just looks at the computer and goes that way, and said, 'What's wrong with you ah? Why no control ah? You eating too much?' [Mimics an impatient and brash tone], you know, that wall IMMEDIATELY gets built up, higher and thicker." (C1)

"I think it would be useful for doctors to show empathy and that may come with training because not everybody has that personality." (B4)

"Our local doctors - and here I am talking about maybe, Chinese doctors (compared to) non-Chinese doctors, the young ones are very arrogant. The older ones just want to scold you, you know, the minute they see that your blood sugar is high, they just go on and scold you and scold you and scold you, without understanding the other circumstances happening to you over the last three months." (C4)

The second attitude was the doctor's encouragement. If friendliness helped to break down the walls of communication, encouragement seemed to lift patients to more enablement. Heartfelt praise for patients when they did well translated into feelings of joy, which spurred patients to do even better in their condition at the next visit.

"My own personal experience is senior ones tend to give you more advice. Of course, they are also encouraging, tap your shoulder and say well done, very encouraging." (B2) "And then she said, 'At least I taught you once and you know how to do it. A lot of elderly people, I teach them so many times, still can't, unable to do it.'" (C3)

The third attitude was doctor's concern. Probing of a condition was seen to be an indication that the doctor was keen to find out more about the condition, as patients would not know which part of the history is important in clinical reasoning. Doctors who emphasised aspects that were severe and advised patients to take their illness seriously were often successful in making an impact in the patient's mind that they needed to manage their condition well. Conversely, doctors who did not convey the severity of a condition was deemed to be uncaring, if they did not emphasise a patient's poor control or abnormal laboratory tests.

"You have to be very, very careful, take this illness very seriously.' I was quite taken in by what she has told me, so I know how serious it is." (J027)

"'Oh then you must be careful, because it's a silent killer.' Then from there, I'm eager to know what type of silent killer is that." (A2)

\section{Theme 3: Doctor-patient relationship Harmony and personalising care}

This theme was related to a doctor's attitude and bearing, but was seen more as an outcome of relationship building that a doctor was able to effect. For example, if a doctor had been friendly and concerned, but a patient was still not keen to put into practice what he had heard, then the patient would not be enabled to manage his condition. 
"Because on my part, I definitely have to do something, if the doctor say and I don't do, we need both parties to cooperate, doctor's advice and we do on our part." (B2)

Patients appreciated doctors who customised their care and advice to their individual situation. Sometimes, it meant taking more time to explain and speak to elderly patients because they seemed slower to understand medical information due to their age. An example was dietary counselling, where an Indian patient was given generic information regarding porridge and certain vegetables that were not culturally a staple food of the Indians. Another example of individualising treatment was how a doctor allowed a patient to self-titrate his insulin doses based on the patient's ability and responsibility to judge his carbohydrate intake and monitor his glucose level. Staff should be able to customise patient education based on how long a patient had been diagnosed with a condition, as newly diagnosed patient would need to know information that is different from patients who are uncontrolled and had been struggling for many years.

"It's like, whether you have one-year-old diabetes and ten years' diabetes, you have gone through different experiences. So you cannot treat those who had 10-20 years the same as those who just got diabetes." (B4)

\section{Theme 4: Continuity of care}

Follow through, post-consult checks and team-based care Enablement was enhanced when patients continued to see the same doctor that they had a rapport with. A certain level of understanding and familiarity seemed to be important, even when patients acknowledged that the same doctor would not remember all that had happened over the last few consultations. However, they felt that at least some aspects may be triggered and be helpful in managing their condition. Participants felt that even though medical details could be recorded in the electronic medical records for follow-up by another doctor, it would still not be the same, as some doctors may not be as comprehensive in looking through a patient's record to know what treatment had been tried and failed. Also, different doctors may communicate and practice differently, which could frustrate or confuse patients' understanding of their therapy. Most importantly, some patients became apathetic and refused to share more with an unfamiliar doctor, which could lead to detrimental effects on that doctor's understanding of the patient's condition.

"The problem is, I won't see him again ... He understood my problem. Now the problem is, I am going to see another doctor. This is exactly what I mind. So it's the same doctor, of course he would not be able to remember everything, because he will be seeing hundreds and hundreds of patients. If you highlight a little bit and the thing will kick in, he will try to remember what he has said. So when I go and see another doctor, the doctor doesn't know anything about it." (C1)

"It also depends on the doctor. It depends on how in-depth they really flip through your past record, whether continue the same advice, also depends on the doctor." (C3)

Patients appreciated phone calls from healthcare staff to find out if they were managing well. A common example was how healthcare assistants would call patients who were discharged from hospitals before their primary care appointments, so as to determine if the patients were adhering to treatment. Such checks allowed patients to clarify any doubts and made them feel that healthcare staff have put in effort to ensure they were recovering. Currently, in the local polyclinic setting, there are only reminders for appointments for certain services, but not all.

"I mean, they really have a system where they don't discharge you just like that. The thing is, they keep following up on all this thing. (J45)

Participants appreciated team-based care in the clinic, where allied health personnel such as dieticians and physiotherapists could be engaged on an as-needed basis. This helped them to fill in gaps in knowledge, especially since doctors were understood to be busier and were unable to spend adequate time to explain certain aspects of care to them.

"I don't think doctors have the time to tell them what to do, what to eat, what to do this thing, but the doctors can refer them to dietician, or anybody who can explain to them the better care." (J34)

\section{Theme 5: Image of setting}

Participants had the impression that they could trust a clinic if they felt that it had a good image. Besides word-of-mouth, some patients felt that the comprehensive services available at a polyclinic setting helped them to better manage care, as they did not need to travel to multiple places in order to do the various investigations required. However, with regard to therapy, most participants also had the impression that the medications that were available at the polyclinic were not as effective compared to that provided by private GPs, as they were subsidised.

"Because the polyclinic comes with a whole slew of support systems and.... So that has been very helpful. It's almost like a holistic approach to manage my health condition. Whereas if I go to GP, even if I had to go X-ray I go SATA (Singapore Anti-Tuberculosis Association), so the process.... At polyclinic, you may wait for three hours or four hours, but if you go to GP, a few days wasted, you go run here, run there, that kind of thing. So in that sense, the polyclinic system, we may have a lot of complaints, has been quite helpful." (B4)

"But one thing, poly(clinic) limited kind of medicine. Poly(clinic) medicine is always lower grade. Sorry to say that... I go to my regular GP for my flu, they got good medicine. But sometimes when you come to poly(clinic), and you want to ask for it, they don't have." (B1)

\section{Theme 6: Workload}

Participants were cognisant that in the public health setting (i.e. polyclinics), healthcare staff suffered from chronic overload. This resulted in them often rushing through a case and thus not delivering care personally. Some patients also felt bad if they took up too much of a doctor's time.

"Because if doctors are busy... someone told me there is a quota that they must see 20 patients or 30 within that time 
(frame)? Then if they talk too long to the patient, they don't have the time to finish or attend to the other (patients)." (J12)

\section{Theme 7: Financial considerations}

Participants reflected that after the diagnosis, the cost of managing their condition can sometimes become quite high, especially if they were being followed up by a private GP. In addition, regular screening tests such as diabetic retinal photography and diabetic foot screenings would cost double at the GP as compared to the service provided by the polyclinic; thus, patients would avoid going for such screenings altogether, which would affect the management of their condition. In terms of consumables, participants recounted that in some countries in Oceania, patients are highly subsidised for glucose strips, and that has helped them to monitor their condition better and more frequently.

"But again the problem comes in, cost is another factor. I have a brother who is in New Zealand, he is also diabetic but very early stage. What he tells me is that when he goes for check-up, the doctors give them the monitoring. So all that is given at highly subsidised rate. He hardly pays anything. That is also important. Because when you buy, you pay something like almost 20 dollars, and it works out to almost a dollar for one stick. And you are going to monitor very frequently, it's going to be quite expensive. And most diabetic patients are usually elderly, probably they are retired, cost also becomes a factor." (C4)

\section{Theme 8: Passive information}

Participants welcomed posters, pamphlets or even the availability of Internet websites as convenient sources of information, and also as sources of new information which they did not know of. However, sometimes they would become less enabled if there was a deluge of information, or if the information was contradictory in nature.

"(For) certain things like, if (the polyclinic) got something on and they can paste on the wall, at least people come to know, 'Oh! You got (this)!', and if you want a certain service. Then at least, some (people who) can read, can (come to know about it)." (J12)

"Like, certain thing, you are not supposed to eat. Then a little later, you can eat, that is good for you. These kind of contradictory things, you see? Like, they say, 'You don't take sugar. But you can take palm sugar, because it's low cholesterol.' So, sugar is (still) sugar what? Then, coconut, all (parts of it is) no good at one time. Now, coconut oil (is) very good." (J34)

\section{Theme 9: Patient's attitude}

\section{Beliefs and mental conditioning}

Patients felt that they were the final arbiter of their disease and its impact on their bodies. Thus, some mentioned that they would trust themselves and decide what advice to follow and what not to. Others spoke about patients who had pessimistic outlooks of their disease and felt that nothing could be done to improve it. These attitudes sometimes impaired enablement, as the patients would decide not to follow medical advice. In the Asian context, traditional or complementary forms of medicine abound. Thus patients often made their own judgements on whether to follow what is prescribed by a doctor or what is prescribed by a traditional medicine practitioner. Concepts of 'heatiness' of a condition and traditional remedies were brought up by patients, as Western medicines were perceived to be generally more 'heaty'. "For me, I am a bit stubborn, you tell me not to eat, like that three times, at least I eat two times. That is my own stubbornness. Because to me, life is short. What I can do, I do. As long as all in my ... I know what not to overtake, not overdo. But I still do." (B1)

"So then probably Chinese physician says ... very 'heaty', things like that ... so probably you need the Chinese medication ... it helps." (B2)

"When you talk about sprains, probably doctor can only give painkiller. But the actual treatment, physio(therapy), traditional masseurs, actually has helped. So I guess over the years, I begin to learn what type of ailments you go where, in that sense." (B4)

Patients who were prepared to manage their conditions often reached that state after being prepped beforehand. Examples of preparatory settings included lectures given by nurses for disease education or information given by healthcare staff when patients were diagnosed with their conditions.

"There was a talk by a nurse, a group of people who were given some lecture by some nurses, and this nurse said that if you have diabetes for about 20 years or more, then they call it 'burn out', then you might have to take insulin to get better results. So mentally, it is already conditioned that ... when I took insulin, (as) I already had diabetes for more than 20 years. So mentally, I was already prepared." (B1)

\section{Inquisitiveness and self-motivation}

Patients felt that they needed to play their part and ask questions when they were unsure. They knew that if they did not request for information, it would be detrimental to their management.

"Actually, I personally feel is, the patient must ask. And the patient also must be honest to tell the doctor the precise situation, rather than hide, because anything that you hide, may not lead to proper conclusion or diagnosis." (A1)

Most participants felt that a high degree of self-motivation was critical. They knew that most patients did not want to be sick but to be well, and did not want to be 'dependent' on taking medications 'all their lives'. However, they knew it required more than just desire, but also willpower and a serious determination to improve.

"I think for diabetes, even doctor advises you or gives you medicine, it won't really help. You have to help yourself. I mean, willpower is very important for a person." (B1)

\section{Trust}

Participants felt that they would trust a doctor's comments about their condition and medications. Whenever there was conflicting health information that was found on the 
Internet, patients tended to seek out the opinions of doctors as professionals.

"I think it still comes from the doctor. They are like professionals. It would be helpful if doctors could advise on some of the side effects, rather than we go and google. All kinds of funny things start coming out... So you ask me the source of ... It should still be coming from doctor or professional." (B2)

\section{Theme 10: Impact of initial care}

Participants who had been told of the impact of their disease in no uncertain terms at the beginning stages of diagnosis typically remembered such advice better.

\section{Consequences and success}

Participants who had been notified of the possible consequences of poor control of their chronic illness often became more interested in their own health. Sometimes, these consequences had to be driven home using pictures. Patients who had actually 'suffered' in the hospitals due to their disease remembered the experience and were determined not to let their health deteriorate lest they had to be admitted for the same condition again.

"It was because of the hospital stay that makes me really suffer." (J45)

"Especially if it is diagnosed for the first time ... that it is very important the patient be told about the severity of a problem if you are not careful." (J27)

Success after initial care helped to boost patients' confidence and enabled them to manage better. Participants remarked that after receiving good advice from healthcare staff, along with plenty of encouragement, the final test to show whether the advice was sound would be how much improvement they gained, whether based on objective targets or subjective feelings.

"I think it has to be proven, in that sense, meaning that when I see a doctor right, I get well, I get the result I want, and that is very important to me or to any patient." (J42)

\section{Theme 11: Community}

The image of a clinic and its impact on a patient's trust has been mentioned. Besides that, word of mouth by family and friends outside of a support group setting also helped a patient's enablement. Many participants mentioned that they tended to seek out comments from their close friends to see if there was concordance between what was taught by other doctors and their own doctors, and this would have greater weightage when they were deciding whether to follow certain advice. Interactions with other patients who had similar conditions and more experience with managing the conditions also helped participants learn practical steps to enable themselves. Patients also mentioned that after they had been enabled, they became advocates for their family members by being the source of information and encouragement for their loved ones.

"One session that I found useful and impactful was diabetes patients come together and discuss, discussion session... there were some who had been having diabetes for decades. And then they share about their lifestyle, what they do, and that they are able to manage it. And the impact seems to sink in better." (B4)

\section{Theme 12: Patient's actions}

Participants agreed that after they had been sufficiently motivated to manage their own conditions, they had to take practical steps to improve. Taking and recording their diet history, blood pressure and glucose levels were some examples of self-management.

"Even doctors told me money cannot buy everything, because we have to treasure our own life. We have to play our part." (B1)

\section{DISCUSSION}

Our study found a mean PEI score of 4.5. This is similar to that (mean $4.65 \pm 2.76$ ) reported in a Hong Kong study by Lam et al. ${ }^{(23)}$ However, most studies from the United Kingdom (UK) found lower PEI scores. ${ }^{(3)}$ In the present study, $64.7 \%$ of the participants were more than 60 years old. Elderly patients tended to have lower PEI scores than younger patients (median 3 [IQR $0-7]$ vs. 5 [IQR 0.5-10]; $p=0.06$ ). Other studies have found that older patients tended to rate enablement more highly. ${ }^{(14)}$ One third of our participants $(n=47)$ rated ' 0 ' for the PEI, which seems to indicate that many patients were not enabled after their consultation. However, according to Brusse and Yen ${ }^{(4)}$ and Lam

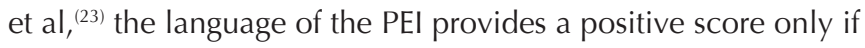
a patient had been more enabled compared to the period before that day's consultation. Thus, well-controlled patients who had been counselled previously may rate the encounter as ' 0 ' (Fig. 2), which creates false-negative scores. There is a need to further develop this tool such that it is better able to differentiate patients who are more enabled from those who are already enabled. ${ }^{(2,4)}$

Patients who were seen at the FPC also rated the PEI significantly higher than patients who were seen at general clinics or nurse-led clinics. In the FPC, where patients saw the same doctor for their conditions, the PEI score was significantly higher than that in the general clinics. Conversely, patients who were seen by the same nurse at nurse-run clinics rated their PEI scores lower than those seen at the other two clinics. Patients seen at nurse-run clinics are chosen by doctors based on a set of criteria and managed via standardised protocols, and are usually well-controlled and thus enabled, leading to no further improvement in PEI scores.

Fig. 2 illustrates a concept map of the three key categories of factors associated with patient enablement found in this study. Factors associated with healthcare staff, especially doctors, had the most impact on patient enablement. Patients responded best to doctors who were friendly, encouraging and able to weave in messages that conveyed the severity of the condition to their patients. Non-verbal communication such as good eye contact was also highlighted in our study. The ability to personalise care based on a patient's life stage, understanding, language and personality was critical to achieving a relationship that is mutually beneficial, as not all patients appreciated information that was unsolicited.

These findings are similar to those reported in other studies. Some studies have noted that empathy, being 'positive' and 


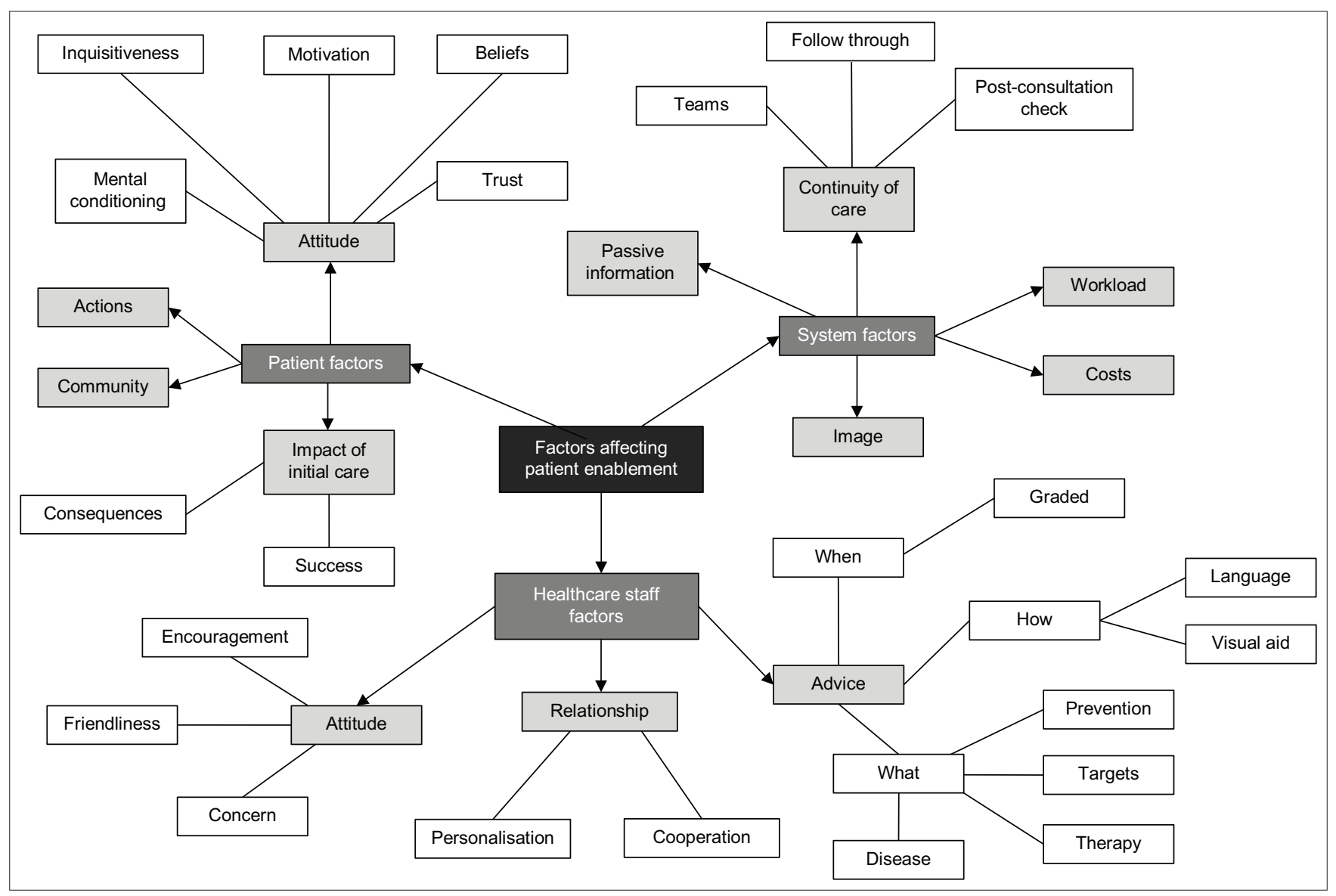

Fig. 2 Concept map shows the factors affecting patient enablement.

having good listening skills were antecedents and key elements of enablement. ${ }^{(11,13,21,29)}$ Task-specific behaviour, such as giving specific disease or therapy advice, has similarly been noted to help patients better manage their conditions. ${ }^{(1)}$ One of the reasons for low enablement is poor information exchange between doctors and patients. An interview of patients after an education intervention found that the information presented was often lacking in importance to the patients. ${ }^{(27)}$ If information that is relevant to a patient could be adequately sought out and presented, patients would likely become more committed to and own their disease. However, doctors tend to be more task-oriented and keener on settling the medical issue in order to deliver evidence-based treatment, rather than exploring the patient's perspective so as to build a good doctor-patient relationship. ${ }^{(30,31)}$

Another aspect that has not been well demonstrated is doctor's attitude of concern. As the adage goes, "People do not care how much you know, until they know how much you care"; patient enablement was noted to be higher if the physician showed interest in the effects of treatment on the patient's life and maintained a positive approach to healthcare. (7) Good doctor-patient communication is an important aspect of the medical consultation, as it helps a physician to come to a right diagnosis, thereafter enhancing the compliance of the patient. Therapeutic effects are seen on biopsychological aspects such as a patient's blood pressure, blood sugar or anxiety level. ${ }^{(32)}$ There are many components of good communications such as asking, listening, praising, advising and checking of understanding or misunderstanding of information. ${ }^{(32)}$ However, it is often difficult to apply these principles in clinical practice due to heavy workload, complex or difficult patients, financial considerations and statutory regulations. ${ }^{(33)}$

A new finding from this study was that patients felt that the use of visual aids helps them to understand their condition better, and that demonstration kits enabled them to learn the skills needed to handle various instruments. Another element of good patient enablement was a graded education plan. For example, patients felt that education on diabetes mellitus delivered at one sitting should not encompass too many aspects of the disease pathology and therapeutics. Skills in delivering advice in a graded fashion can be taught to junior doctors directly or indirectly when they sit in with senior doctors. ${ }^{(34)}$

Continuity of care has been highly regarded as a critical factor in patient enablement. However, only $38.4 \%$ of Singaporeans have been noted to see the same regular family physician. ${ }^{(35)}$ Our study reinforces the need for patient to have a regular doctor, as patients who saw the same doctor rated higher enablement than those who did not. This is similar to the findings of other studies. ${ }^{(13,25,34)}$ Practically, a system of follow-up for patients with the same doctor or team, especially for those who have been started on new medications or treated for asthma exacerbations, would potentially improve patient enablement.

System factors, such as the capability of polyclinics to provide a wide array of services to patients with chronic conditions, have 
been regarded as important for patient enablement. In Asia, Hong Kong has leveraged on polyclinics as a supportive setup for hospitals, so as to provide more accessible care to patients who reside further away from hospitals. Such polyclinics may be a subsidiary of the main hospitals (e.g. Union Hospital in Hong Kong) and can also be equipped with radiology and laboratory services. ${ }^{(36)}$ If certain services such as laboratory or radiology services are not easily available, it would be more difficult for patients to manage their conditions. Practically, convenience and co-location of resources are thus deemed to be important facets of patient enablement. These considerations have also been shown to be important to a patient when deciding whether to attend a polyclinic. ${ }^{(37)}$

In most Asian primary care systems, access to medical care is not a problem, as most patients are still able to consult a doctor without a pre-fixed appointment. Most Western healthcare setups have a more elaborate system of phone triage before patients are scheduled to see a doctor or trained nurse. The workload of a clinic has an inverse relationship with the amount of time and effort that can be spent on each patient. In the Asian context where consultation times are generally shorter, ${ }^{(38)}$ it is typically more difficult for healthcare providers to delve deeply into the biopsychosocial aspect of a patient. ${ }^{(39)}$ An exception to this is the FPCs, where more time is catered.

The cost of care was also highlighted in this study, which was not examined in other studies. Other Asian countries, such as Korea, Japan and Taiwan, rely less on a system of governmentsubsidised polyclinics and more on social health insurance to pay for healthcare expenses. ${ }^{(40)}$ Studies from non-Asian countries have not explored the relationship between financial burdens and patient enablement. Only Leeseberg Stamler et al mentioned that a patient's means, together with the opportunities and abilities to manage their care, was important for enablement. ${ }^{(27)}$

Patients who suffered from complications of disease or were cognisant of the consequences of poor control seemed keener to manage their disease better. This aspect of patient enablement has not previously been brought up; most conceptual analyses of patient enablement have highlighted its antecedents and attributes, but no study has mentioned the effects of prior experiences on enablement.

In this study, the background and makeup of patients played an important role in enablement. Some patients who had been previously conditioned to accept changes in their disease were more able to cooperate, while those with fixed mindsets would not accept the doctor's advice. In a study involving Polish patients, Pawlikowska et al reflected: "There is evidence that Polish patients not only feel their doctors are important, but trust in doctors remains high and Polish patients 'viewed medicine as a useful tool ... but felt they had responsibility for their own health; so patients may enter the consulting room in a frame of mind that facilitates enablement". (20) In the aforementioned study, the Polish patients had a higher mean PEI score compared to UK patients (4.0 vs. 3.1). Being positive, self-motivated and inquisitive are thus important qualities that improve patient enablement. This is also another aspect of patient-centred care that has not previously been highlighted, as most studies have concentrated on the impact of a patient's age, case mix and psychological issues. ${ }^{(9)}$ Hudon et al's study mentioned antecedents of enablement such as 'positive outlook and acceptance of the person', as well as attributes such as 'consideration of the person as a whole'. ${ }^{(3)}$

This study also brought out the importance of the community in promoting enablement. Community input helped patients see beyond their current state, when they witnessed older patients or those who had had the condition for a longer time manage their disease and overcome them. We postulate that Asian communities may tend to be more communal, and thus, the comments and advice from other patients are more respected and imbibed. Practically, clinics could leverage on patients who have good control over their conditions and enlist them either as 'champions' of care to encourage other patients to do better, or create support groups that provide practical tips on how to be healthy while dealing with a disease. Indeed, at our FGDs, some patients were seen to encourage others to do home glucose monitoring or enrol into FPCs so that they could enjoy better health.

This study was not without limitations. In the quantitative phase of the study, patients were approached as they were leaving the consultation rooms and heading toward the pharmacy. Thus, convenience sampling had to be employed, as only patients who were keen or able to spend time with the researcher would be sampled. ${ }^{(26)}$ To reduce such bias, researchers were trained to follow a protocol when they approached the patients. With more time and manpower, a larger sample size from other healthcare settings would reduce sampling bias. Even though the research setting is noted to be representative of the national patient portfolio and thus returned a PEI that was reflective of the research question (i.e. how patients scored their enablement experiences), the factors associated with enablement (i.e. the second qualitative research question) could have been different for different practice settings.

Many patients in our study recorded PEI scores of ' 0 ' even though they were able to manage their condition. This could have affected the validity of the modality of patient enablement that was being measured. Patients who scored ' 0 ' may have been on follow-up for the condition for many years and would thus have fewer gaps in knowledge or understanding than patients who had been newly diagnosed, and therefore, there would not be any net change in enablement for subsequent visits. ${ }^{(4)}$ In view of this, some authors have done a factor analysis of the six items in the PEI and chosen the items that are most reflective of enablement and integrated it with other questionnaires, ${ }^{(25,41)}$ although the earliest studies had noted that internal consistency of the PEI was reduced if any single item had been removed. ${ }^{(42)}$ More exploration could be done on such relative changes in enablement.

Our study was based on a single primary care clinic. Firstly, we should study a larger sample size. This would reduce sampling bias and afford a stronger inference about the link between PEI and the various patient characteristics. An extension of the sample to include other polyclinics and private GP clinics, or even other surrounding Asian countries, would provide a more holistic picture of patient enablement. Also, future studies could 
stratify participants according to their disease duration to see if there is any reduction in the PEI scores for those who have had the condition for a longer period, since they would have garnered more information and skills over time. An association between PEI scores and outcomes of high enablement (such as control of chronic condition), could help provide some clues as to whether patients with higher PEI scores also have better disease control.

The current study has revealed additional factors that may affect patient enablement, such as the consequences of previous experiences of the disease, the use of good explanation aids, and the capabilities of the healthcare setting. These individual factors should be further explored to determine their impact on enablement. Most previous studies have focused on healthcare staff's behaviours and skills, and future research could concentrate more on systemic factors and the healthcare context of a patient's consultation.

In conclusion, this study has shown that the PEI score in the Singapore context is similar to that among Asian cohorts, although the Asian experience of patient enablement is limited, while it is slightly higher than that recorded in Western studies. Participants who were managed in more specialised clinics seem to rate enablement higher. There was no significant difference in PEI scores when comparing age, gender, ethnic groups, education or numbers of medical problems. Conceptually, patients need to possess the knowledge and skills to manage their conditions, ${ }^{\left({ }^{8}\right)}$ as well as the confidence and commitment to improve. Beyond that, patients in Singapore are affected by the consequences of their disease and rely on the input of their community to be enabled. System factors such as the availability and affordability of healthcare are key factors affecting enablement of local patients. In addition to good communication skills and doctor-patient relationships, our study has also shown that the use of visual aids and demonstration kits helps to improve a patient's knowledge and understanding.

\section{ACKNOWLEDGEMENTS}

We acknowledge Prof Cees Van Der Vleuten for his invaluable guidance from the conception of this topic till the completion of this research project. His encouragement and wisdom motivated, activated and enabled us to continue this work. We would also like to acknowledge the research team from SingHealth Polyclinics, namely Dr Tan Ngiap Chuan, Ms Caris Tan Yang Thong, Ms Eileen Koh Yi Ling and Ms Usha Sankari, for their technical and financial guidance. We thank clinic director Dr Jonathan Ting Sing Shing from SingHealth Polyclinics - Geylang and his management team for granting us permission to conduct the IDIs and FGDs beyond clinic operating hours. This project would not have been possible without the help of a dedicated team of like-minded colleagues, namely Dr Shah Mitesh, Dr Andrew Fang, Dr Liu Sha and Ms Xu Huiyan, who helped tirelessly with the recruitment of patients amidst their heavy schedules.

The SingHealth Centralised Institutional Review Board $\mathrm{E}$ approved the above research project to be conducted in SingHealth Polyclinics - Geylang (reference: 2016/3019). The study was funded by SingHealth Seed Funding (Reference: P9SEED371606).

\section{REFERENCES}

1. Mead N, Bower P, Hann M. The impact of general practitioners' patientcentredness on patients' post-consultation satisfaction and enablement. Soc Sci Med 2002; 55:283-99.

2. Institute of Medicine (US) Committee on Quality of Health Care in America. Crossing the Quality Chasm: A New Health System for the 21st Century. Washington DC: National Academies Press (US), 2001: 61-88.

3. Hudon C, St-Cyr Tribble D, Bravo G, Poitras ME. Enablement in health care context: a concept analysis. J Eval Clin Pract 2011; 17:143-9.

4. Brusse CJ, Yen LE. Preferences, predictions and patient enablement: a preliminary study. BMC Fam Pract 2013; 14:116

5. Haughney J, Cotton P, Rosen JP, et al. The use of a modification of the Patient Enablement Instrument in asthma. Prim Care Respir J 2007; 16:89-92.

6. Howie JG, Heaney DJ, Maxwell M, Walker JJ. A comparison of a Patient Enablement Instrument (PEI) against two established satisfaction scales as an outcome measure of primary care consultations. Fam Pract 1998; 15:165-71.

7. Andén A, Andersson SO, Rudebeck CE. Satisfaction is not all--patients' perceptions of outcome of general practice consultations, a qualitative study. BMC Fam Pract 2005; 6:43.

8. Fumagalli LP, Radaelli G, Lettieri E, Bertele' P, Masella C. Patient Empowerment and its neighbours: clarifying the boundaries and their mutual relationships. Health Policy 2015; 119:384-94.

9. Mercer SW, Jani BD, Maxwell M, Wong SY, Watt GC. Patient enablement requires physician empathy: a cross-sectional study of general practice consultations in areas of high and low socioeconomic deprivation in Scotland. BMC Fam Pract 2012; 13:6.

10. Mercer SW, Reilly D, Watt GC. The importance of empathy in the enablement of patients attending the Glasgow Homoeopathic Hospital. Br J Gen Pract 2002; 52:901-5.

11. MacPherson H, Mercer SW, Scullion T, Thomas KJ. Empathy, enablement, and outcome: an exploratory study on acupuncture patients' perceptions. J Altern Complement Med 2003; 9:869-76.

12. Little P, Everitt H, Williamson I, et al. Observational study of effect of patient centredness and positive approach on outcomes of general practice consultations. BMJ 2001; 323:908-11.

13. Birhanu Z, Woldie MK, Assefa T, Morankar S. Determinants of patient enablement at primary health care centres in central Ethiopia: A cross-sectional study. African J Prim Heal Care Fam Med 2011; 3:1-8.

14. Howie JG, Heaney DJ, Maxwell M, et al. Quality at general practice consultations: cross sectional survey. BMJ 1999; 319:738-43.

15. Ozvacić Adzić Z, Katić M, Kern J, et al. Patient, physician, and practice characteristics related to patient enablement in general practice in Croatia: cross-sectional survey study. Croat Med J 2008; 49:813-23.

16. Cocksedge S, Greenfield R, Nugent GK, Chew-Graham C. Holding relationships in primary care: a qualitative exploration of doctors' and patients' perceptions. BrJ Gen Pract 2011; 61:e484-91.

17. Banerjee A, Sanyal D. Dynamics of doctor-patient relationship: a cross-sectional study on concordance, trust, and patient enablement. J Family Community Med 2012; 19:12-9.

18. Pawlikowska TR, Nowak PR, Szumilo-Grzesik W, Walker JJ. Primary care reform: a pilot study to test the evaluative potential of the Patient Enablement Instrument in Poland. Fam Pract 2002; 19:197-201.

19. Rolfe A, Cash-Gibson L, Car J, Sheikh A, McKinstry B. Interventions for improving patients' trust in doctors and groups of doctors. Cochrane Database Syst Rev 2014; (3):CD004134.

20. Pawlikowska TR, Walker JJ, Nowak PR, Szumilo-Grzesik W. Patient involvement in assessing consultation quality: a quantitative study of the Patient Enablement Instrument in Poland. Health Expect 2010; 13:13-23.

21. Mercer SW, Reynolds WJ. Empathy and quality of care. Br J Gen Pract 2002; 52 Suppl:S9-12.

22. Yu FS, Yip BH, Kung K, et al. The association of types of training and practice settings with doctors' empathy and patient enablement among patients with chronic illness in Hong Kong. PLoS One 2015; 10:e0144492.

23. Lam CL, Yuen NY, Mercer SW, Wong W. A pilot study on the validity and reliability of the Patient Enablement Instrument (PEI) in a Chinese population. Fam Pract 2010; 27:395-403.

24. Kelly M, Egbunike JN, Kinnersley P, et al. Delays in response and triage times reduce patient satisfaction and enablement after using out-of-hours services. Fam Pract 2010; 27:652-63.

25. Mead N, Bower P, Roland M. Factors associated with enablement in general practice: cross-sectional study using routinely collected data. $\mathrm{Br}$ J Gen Pract 2008; 58:346-52

26. Creswell JW. Educational research: Planning, conducting, and evaluating quantitative and qualitative research. Vol. 3, Educational Research. 2012.

27. Leeseberg Stamler L, Cole MM, Patrick LJ. Expanding the enablement framework and testing an evaluative instrument for diabetes patient education. J Adv Nurs 2001; 35:365-72

28. McHorney CA, Tarlov AR. Individual-patient monitoring in clinical practice: are available health status surveys adequate? Qual Life Res 1995; 4:293-307. 
29. Hudon C, St-Cyr Tribble D, Bravo G, et al. Family physician enabling attitudes: a qualitative study of patient perceptions. BMC Fam Pract 2013; 14:8.

30. Arborelius E, Timpka T. Comparison of patients' and doctors' comments on video-recorded consultations. Scand J Prim Health Care 1991; 9:71-7.

31. Agledahl KM, Gulbrandsen P, Førde R, Wifstad $\AA$. Courteous but not curious: how doctors' politeness masks their existential neglect. A qualitative study of video-recorded patient consultations. J Med Ethics 2011; 37:650-4.

32. Shendurnikar N, Thakkar PA. Communication skills to ensure patient satisfaction. Indian J Pediatr 2013; 80:938-43.

33. Kuehl SP. Communication tools for the modern doctor bag. Physician patient communication part 1: beginning of a medical interview. J Community Hosp Intern Med Perspect 2011; 1(3)

34. Wensing M, Jung HP, Mainz J, Olesen F, Grol R. A systematic review of the literature on patient priorities for general practice care. Part 1: description of the research domain. Soc Sci Med 1998; 47:1573-88.

35. Ministry of Health, Singapore. National Health Surveillance Survey 2007. Available at: https://www.moh.gov.sg/content/moh_web/home/Publications/Reports/2009/ national_health_surveillancesurvey2007.html. Accessed April 1, 2017.
36. Union Hospital. Services: Polyclinics. Available at: https://www.union.org/new/ english/services/services_polyclinics.htm. Accessed June 30, 2018.

37. Chow WL, Wang VW, Low YS, Tse DW, Lim JF. Factors that influence the choice of seeking treatment at polyclinics. Singapore Med J 2012; 53:109-15.

38. Claramita M, Utarini A, Soebono H, Van Dalen J, Van der Vleuten C. Doctorpatient communication in a Southeast Asian setting: the conflict between ideal and reality. Adv Heal Sci Educ Theory Pract 2011; 16:69-80.

39. Mead N, Bower P. Patient-centredness: a conceptual framework and review of the empirical literature. Soc Sci Med 2000; 51:1087-110.

40. Leung GM, Bacon-Shone J. Hong Kong's Health System: Reflections, Perspectives and Visions. Hong Kong University Press, 2006.

41. Kurosawa S, Matsushima M, Fujinuma $Y$, et al. Two principal components, coping and independence, comprise patient enablement in Japan: cross sectional study in Tohoku area. Tohoku J Exp Med 2012; 227:97-104.

42. Howie JG, Heaney DJ, Maxwell M, Walker JJ. A comparison of a Patient Enablement Instrument (PEI) against two established satisfaction scales as an outcome measure of primary care consultations. Fam Pract 1998; 15:165-71. 